\title{
A NEW PROOF OF THE GRAUERT DIRECT IMAGE THEOREM
}

\author{
R. LEVY
}

\begin{abstract}
A new proof of the Grauert direct image theorem based on methods of functional analysis is given.
\end{abstract}

Let $f: M \rightarrow N$ be a proper morphism of complex spaces, and let $\mathcal{L}$ be a coherent sheaf on $M$. The generalized direct images $R^{p} f_{*} \mathcal{L}$ are the sheaves on $N$ associated with the presheaves $V \rightarrow H^{p}\left(f^{-1}(V), \mathcal{L}\right)$. One denotes by $f_{!} \mathcal{L}$ the complex consisting of sheaves $R^{p} f_{*} \mathcal{L}$ and having zero differentials. Grauert's direct image theorem (see [1]) asserts that all the sheaves $R^{p} f_{*} \mathcal{L}$ are coherent on $N$. Our aim is to give a proof of the theorem for readers more familiar with functional analysis than with the theory of complex spaces. There are two main differences between the present proof and that of Forster and Knorr [2]. First, we give a canonical construction, perhaps simpler, of a resolution of the direct image. Second, the coherence of the homology sheaves of this resolution is obtained as a corollary of some general facts concerning parameterized complexes of Fréchet spaces, which may be of independent interest.

In $\S 1$ we construct a resolution of the direct image by infinite-dimensional free sheaves-i.e., by sheaves of germs of holomorphic vector-functions with values in a Fréchet space. The construction is based on the notion of a parameterized Koszul complex for a commuting $n$-tuple of operators introduced in $[\mathbf{3}]$. Note that the construction is in some sense canonical (see $\S 4$ ). The other main step in the proof is a parameterized variant of the $\mathrm{L}$. Schwartz perturbation theorem (Lemma 3.1). We use this assertion in the same manner as the classical Schwartz theorem is used in the Cartan-Serre proof of the finiteness theorem. An important step in the proof of Lemma 3.1 is Lemma 2.1, which asserts that the lifting for any parameterized complex that admits a lifting of holomorphic sections can be done with uniform estimates. Applying Lemma 3.1 to the infinite-dimensional resolution constructed in $\S 1$, we show that this resolution is quasi-isomorphic to a complex of free sheaves of finite type, and therefore all its homology sheaves are coherent, which is the statement of Grauert's theorem.

DEFInition. A holomorphic parameterized complex of Fréchet spaces $X .(\lambda)$ on the domain $U$ is a system $\left\{X_{i}, d_{i}(\lambda)\right\}$, where $X_{i}$ are Fréchet spaces and $d_{i}(\lambda): X_{i} \rightarrow$ $X_{i+1}$ are bounded operators, holomorphically depending on the parameter $\lambda \in U$ and satisfying $d_{i+1}(\lambda) \circ d_{i}(\lambda) \equiv 0$. All complexes will be assumed bounded; i.e. $X_{i}=$ 0 for $|i|$ sufficiently large. We shall denote by $O X$ the sheaf of germs of holomorphic functions with values in the Fréchet space $X$, and by $O X .=\left\{O X_{i}, d_{i}(\lambda)\right\}$ the complex of sheaves of germs of holomorphic sections of the complex $X .(\lambda)$ on $U$.

Received by the editors December 13, 1984 and, in revised form, July 16, 1985.

1980 Mathematics Subject Classification. Primary 46E40; Secondary 32C15.

Key words and phrases. Parameterized complex, Koszul complex, coherent sheaf. 
1. A Koszul resolution of the direct image of a sheaf. In this section we shall use the notions of bicomplex and corresponding total complex. By definition, a bicomplex $\left(E_{i, j}, d_{i, j}^{\prime}, d_{i, j}^{\prime \prime}\right)$ of vector spaces (modules, sheaves) is defined by a system of spaces $E_{i, j}, i, j \in \mathbf{Z}$, and morphisms $d_{i, j}^{\prime}: E_{i, j} \rightarrow E_{i+1, j}, d_{i, j}^{\prime \prime}: E_{i, j} \rightarrow E_{i, j+1}$ satisfying the conditions $d^{\prime} \circ d^{\prime}=0, d^{\prime \prime} \circ d^{\prime \prime}=0, d^{\prime} \circ d^{\prime \prime}+d^{\prime \prime} \circ d^{\prime}=0$. The corresponding total complex is determined by the spaces $E_{n}=\bigoplus_{i+j=n} E_{i, j}$ and differentials $d: E_{n} \rightarrow E_{n+1}$ given by the formula $d=d^{\prime}+d^{\prime \prime}$. Then $d \circ d=0$; i.e., the system $\left\{E_{n}, d\right\}$ is a complex. Now, let $T=\left(T_{1}, \ldots, T_{n}\right)$ be a commuting $n$-tuple of linear operators acting in the Fréchet space $X$. Recall that the parameterized Koszul complex $K .(T, X)(\lambda)$ for the $n$-tuple $T$ in $X$ (cf. $[\mathbf{3}])$ is the holomorphic parameterized complex on $\mathbf{C}^{n}$ equal at the point $\lambda=\left(\lambda_{1}, \ldots, \lambda_{n}\right) \in \mathbf{C}^{n}$ to the Koszul complex for the operators $T_{1}-\lambda_{1} I, \ldots, T_{n}-\lambda_{n} I$ in $X$. Recall that each of the components of the parameterized Koszul complex is a direct sum of finitely many copies of the space $X$, and the differentials are matrices whose entries are linear functions of $T_{1}, \ldots, T_{n}$ and $\lambda_{1}, \ldots, \lambda_{n}$. This construction can be performed in a more general situation. Let $X .(\mu)=\left\{X_{i}, d_{i}(\mu)\right\}$ be a holomorphic complex of Fréchet spaces on the domain $U$, and let $T=\left(T_{1}, \ldots, T_{n}\right)$ be an $n$-tuple of commuting endomorphisms of the complex $X(\mu)$-i.e., the action of $T_{1}, \ldots, T_{n}$ on the spaces $X_{i}$ commutes with the differentials $d_{i}(\mu)$. Then each differential $d_{i}(\mu)$ induces a morphism of complexes $d_{i, \cdot}(\mu): K .\left(T, X_{i}\right)(\lambda) \rightarrow K .\left(T, X_{i+1}\right)(\lambda)$. In such a way we obtain a holomorphic bicomplex $\left\{E_{i, j}, d_{i, j}^{\prime}(\lambda, \mu), d_{i, j}^{\prime \prime}(\lambda, \mu)\right\}$, with $E_{i, j}=K_{i}\left(T, X_{j}\right), d_{i, j}^{\prime}(\lambda, \mu)$ equal to the $i$ th differential in the complex $K .\left(T, X_{j}\right)(\lambda)$, and $d_{i, j}^{\prime \prime}(\lambda, \mu)=(-1)^{i} d_{i, j}(\mu)$. The total complex of this bicomplex will be called the parameterized Koszul complex for $T$ in $X .(\mu)$ and will be denoted by $K X \cdot(T)(\lambda, \mu)$. This construction is functorial; if $\varphi \cdot(\mu): X \cdot(\mu) \rightarrow Y \cdot(\mu)$ is a morphism of parameterized complexes, then it lifts canonically to a morphism $K \varphi \cdot(\lambda, \mu): K X .(T)(\lambda, \mu) \rightarrow K Y \cdot(T)(\lambda, \mu)$.

Let $\mathcal{L}$ be a sheaf of Fréchet $O_{U}$-modules on the Stein domain $U \subset \mathbf{C}^{n}$, let $\mathcal{L}(U)$ be the Fréchet space of all sections of $\mathcal{L}$ over $U$, and let $M=\left(M_{z_{1}}, \ldots, M_{z_{n}}\right)$ be the $n$-tuple of operators given by multiplication with the coordinate functions $z_{1}, \ldots, z_{n}$ on $\mathbf{C}^{n}$ acting in the space $\mathcal{L}(U)$.

LEMMA 1.1. The complex of sheaves $\mathcal{O} K .(M, \mathcal{L}(U))$ is quasi-isomorphic to the sheaf $\mathcal{L}$ on the domain $U$.

Proof. Set $U^{\prime}=U \times U$, and let $p_{1}, p_{2}$ be the projections of $U$ on the first and second components, and let $\mathcal{L}^{\prime}=p_{2}^{*} \mathcal{L}$. Then the sheaf of holomorphic functions on $U$ with values in the Fréchet space $\mathcal{L}(U)$ coincides with the direct image $p_{1 *} \mathcal{L}^{\prime}$ of $\mathcal{L}^{\prime}$. Let $\lambda=\left(\lambda_{1}, \ldots, \lambda_{n}\right)$ and $z=\left(z_{1}, \ldots, z_{n}\right)$ be the coordinate systems on the first and the second copies of $U$, and let $\Delta$ be the diagonal in $U^{\prime}$. Denote by $K .(\lambda-z)$ the Koszul complex for the functions $\lambda_{1}-z_{1}, \lambda_{n}-z_{n}$; then the complex of sheaves $K \cdot(\lambda-z) \rightarrow O_{\Delta} \rightarrow 0$ is exact on $U^{\prime}$. Since the sheaves $\mathcal{L}^{\prime}$ and $O_{\Delta}$ are Tor-independent, then, tensoring this complex by $\mathcal{L}^{\prime}$, we obtain an exact complex $K .(\lambda-z) \otimes \mathcal{L}^{\prime} \mapsto \mathcal{O}_{\Delta} \otimes \mathcal{L} \mapsto 0$ on $U^{\prime}$. Taking the direct image by $p_{1}$, we obtain the exact complex of sheaves $\mathcal{O} K .(M, \mathcal{L}(U)) \rightarrow \mathcal{L} \rightarrow 0$ on $U$.

COROLlary. Let $V$ be a Stein domain in $\mathbf{C}^{m}, \mathcal{L}$ a coherent sheaf on $W=V \times$ $U$, and $p$ the projection of $W$ on $U$. Then the complex of sheaves $O K .(M, \mathcal{L}(W)) \rightarrow$ $p_{*} \mathcal{L} \rightarrow 0$ is exact on $U$. 
Let $S$ be a complex space, $U$ a Stein domain in $\mathbf{C}^{n}, f: S \rightarrow U$ a proper morphism of complex spaces, and $\mathcal{L}$ a coherent sheaf on $S$. Let $\mathcal{U}=\left\{\left(W_{i}, \varphi_{i}, U_{i}\right)\right\}, i \in I$, be an atlas on $S$. This means that the $W_{i}$ are open subsets of $S, U_{i}=V_{i} \times U$, where $V_{i}$ are Stein domains in some $\mathbf{C}^{m}$, and $\varphi_{i}$ are closed embeddings of $W$ in $U_{i}$ such that the restriction of $f$ on $W_{i}$ agrees with the projection of $U_{i}$ on $U$. Denote by $C \cdot(S, \mathcal{U}, \mathcal{L})$ the alternating cochain complex corresponding to the covering $\mathcal{U}$ and the sheaf $\mathcal{L}$ on $S$. If $z_{1}, \ldots, z_{n}$ is a coordinate system on $U$, then the corresponding operators of multiplication $M_{z_{1}}, \ldots, M_{z_{n}}$ act as endomorphisms of the complex of Fréchet spaces $C \cdot(S, \mathcal{U}, \mathcal{L})$. Denote by $K C \cdot(S, \mathcal{U}, \mathcal{L})(\lambda)$ the corresponding parameterized Koszul complex on $U$.

LEMMA 1.2. The complex of sheaves $O K C \cdot(S, \mathcal{U}, \mathcal{L})$ is quasi-isomorphic on $U$ to the generalized direct image $f_{!} \mathcal{L}=R^{*} f_{*} \mathcal{L}$.

PROOF. It follows from Lemma 1.1 that for any open Stein domain $V \subset U$ the complex of sections of $\mathcal{O} K C \cdot(S, \mathcal{U}, \mathcal{L})$ over $V$ is quasi-isomorphic to the complex $C .\left(f^{-1}(V), \mathcal{U}_{f^{-1}(V)}, \mathcal{L}_{f^{-1}(V)}\right)$. Recalling the definition of the generalized direct image, we obtain the assertion of the lemma.

Let the atlas $\mathcal{U}^{\prime}$ be a refinement of $\mathcal{U}$. Then there exists a morphism of refinement $R_{\mathcal{U}, \mathcal{U}^{\prime}}: C \cdot(S, \mathcal{U}, \mathcal{L}) \rightarrow C \cdot\left(S, \mathcal{U}^{\prime}, \mathcal{L}\right)$. Using the functoriality of the construction of the Koszul complexes, we obtain a morphism of parameterized complexes on $U: K R_{U, u^{\prime}}: K C \cdot(S, \mathcal{U}, \mathcal{L})(\lambda) \rightarrow K C \cdot\left(S, \mathcal{U}^{\prime}, \mathcal{L}\right)(\lambda)$. Lemma 1.2 shows that $K R_{U, U^{\prime}}$ induces a quasi-isomorphism from the complex of sheaves $O K C \cdot(S, \mathcal{U}, \mathcal{L})$ to $O K C \cdot\left(S, \mathcal{U}^{\prime}, \mathcal{L}\right)$. Further, let $V$ be a Stein domain, $V \Subset U$. Then the restriction from $U$ to $V$ induces a morphism of complexes

$$
R_{U, V}: C \cdot\left(S, U^{\prime}, \mathcal{L}\right) \rightarrow C \cdot\left(f^{-1}(V),\left.U^{\prime}\right|_{f^{-1}(V)},\left.\mathcal{L}\right|_{f^{-1}(V)}\right),
$$

and a morphism of parameterized complexes

$$
K R_{U, V}: K C \cdot\left(S, \mathcal{U}^{\prime}, \mathcal{L}\right)(\lambda) \rightarrow K C \cdot\left(f^{-1}(V),\left.\mathcal{U}^{\prime}\right|_{f^{-1}(V)},\left.\mathcal{L}\right|_{f^{-1}(V)}\right)(\lambda) .
$$

Again from Lemma 1.2 it follows that this morphism induces a quasi-isomorphism of the corresponding complexes of sheaves of holomorphic sections on $V$. Now consider the superposition $K R_{U, V} \circ K R_{u, u^{\prime}}$. It is easy to see that this morphism induces a quasi-isomorphism of the complexes of sheaves of holomorphic sections, and all components of this morphism are compact operators. In $\S 3$ we show that this implies that all homology sheaves of the complex $O K C \cdot(S, \mathcal{U}, \mathcal{L})$ are coherent, which is the statement of the Grauert theorem.

2. Lifting of sections of exact parameterized complexes. If a given parameterized complex of Banach spaces is pointwise exact on some domain, then the complexes of holomorphic or continuous sections are also exact (see [3]). However, this is not true for parameterized complexes of Fréchet spaces. Because of this we consider here a more special class of exact complexes.

DEFINITION. The holomorphic parameterized complex $X .(\lambda)$ will be called 0 exact if the complex $O X$. of sheaves of germs of holomorphic sections of $X .(\lambda)$ is exact. If we denote by $H(U, X)$ the space of all holomorphic functions on the domain $U$ with values in the Fréchet space $X$, then this is equivalent to the fact that for any Stein domain $U$ the complex $H(U, X$.$) is exact. The morphism of holo-$ morphic complexes $\varphi_{\cdot}(\lambda): X .(\lambda) \rightarrow Y .(\lambda)$ will be called an 0 -quasi-isomorphism if 
it induces a quasi-isomorphism between $O X$. and $O Y$; i.e., if its cone is $O$-exact. A holomorphic complex of Fréchet spaces will be called 0 -Fredholm if on every sufficiently small domain it is $\mathbf{O}$-quasi-isomorphic to a bounded holomorphic complex consisting of finite-dimensional spaces. It is well known that the homology sheaves of a bounded complex, consisting of finite-dimensional free $\mathbf{O}$-modules, are coherent-i.e., roughly speaking, they can be represented by a finite number of generators and a finite number of relations between the generators. Therefore, if $X .(\lambda)$ is $O$-Fredholm, then all the homology sheaves of the complex $O X$. are coherent. It is also easy to prove the converse assertion, but we do not use it in this paper.

It will be necessary for our purposes to consider another more complicated definition of $O$-exactness. For a given polydisk $U \subset \mathbf{C}$ and a Fréchet space $X$, denote by $A(U, X)$ the space of all $X$-valued functions $x(\lambda)$, holomorphic on $U$ and continuous on $\bar{U}$, endowed with seminorms $\|x(\lambda)\|_{U, p}=\sup \|x(\lambda)\|_{p}, \lambda \in U$. Whenever $U$ is a degenerated polydisk (i.e., a point), we put $A(U, X)=X$. The holomorphic complex $X .(\lambda)=\left\{X_{i}, d_{i}(\lambda)\right\}$, defined on the domain $D$, will be called $A$-exact if the following two requirements are satisfied:

(i) For any polydisk $U \Subset D$ the complex $A(U, X$.) is exact. The open mapping theorem shows that in this case for any $U$ and any natural number $p$ there exist a constant $C=C(U, p)$ and a natural number $q=q(U, p)$ such that for any vectorfunction $x(\lambda) \in A\left(U, X_{i}\right)$ satisfying $d_{i}(\lambda) x(\lambda) \equiv 0$ there exists a vector-function $y(\lambda) \in A\left(U, X_{i-1}\right)$ such that $d_{i-1}(\lambda) y(\lambda) \equiv x(\lambda)$ and $\|y(\lambda)\|_{U, p} \leq C\|x(\lambda)\|_{U, q}$.

(ii) For all polydisks $U$ included in some fixed compact subset $K \subset D$ the constants $C(U, p)$ and $q(U, p)$ can be chosen to be independent of $U$.

Note the following property of $A$-exact complexes: if $\lambda_{n} \in K$ is a sequence of points and $x_{n} \in X_{i}$ is a sequence of elements satisfying $d_{i}\left(\lambda_{n}\right) x_{n} \mapsto 0$, then there exists a sequence $u_{n} \in X_{i}$ such that $u_{n} \mapsto 0$ and $d_{i}\left(\lambda_{n}\right) u_{n}=d_{i}\left(\lambda_{n}\right) x_{n}$.

It is easy to see that any $A$-exact complex is 0 -exact. We shall prove the converse statement.

LEMMA 2.1. Let $A$ be a class of holomorphic complexes defined on the polydisk $D \subset \mathbf{C}^{n}$ such that A.

(1) If the differentials of the complex $X$. do not depend on $\lambda$, then $X$. belongs to

(2) If all the rows of a parameterized bicomplex belong to $A$, then the total complex of this bicomplex also belongs to $A$.

(3) If $X$ is a Fréchet space, and $M$ is the $n$-tuple of operators of multiplication by the coordinate functions on $D$ acting in the space $H(D, X)$, then the complex $K .(M, H(D, X))(\lambda) \stackrel{\Delta(\lambda)}{\mapsto} X \rightarrow C$, where $\Delta(\lambda)$ is the evaluation at $\lambda$, belongs to $A$.

(4) If the morphism of complexes $\varphi \cdot(\lambda): X \cdot(\lambda) \rightarrow Y \cdot(\lambda)$ is an A-quasiisomorphism (i.e. its cone belongs to $A$ ), and one of the complexes $X .(\lambda), Y \cdot(\lambda)$ belongs to $A$, then the same is true for the other.

Then $A$ contains all $O$-exact complexes.

ProOF. Let $X \cdot(\lambda)=\left\{X_{i}, d_{i}(\lambda)\right\}$ be an O-exact holomorphic complex on $D$. The complex $H(D, X$.$) is an exact constant complex of Fréchet spaces. Denote$ by $K .(M, H(D, X)).(\lambda)$ the bicomplex consisting of the parameterized Koszul complexes for the $n$-tuple $M$ in the spaces $H\left(D, X_{i}\right)$. (1) and (2) show that the corresponding total complex (denote it by $K X .(\lambda)$ ) belongs to $A$. On the other hand, 
(3) and (2) show that the maps $\Delta(\lambda)$ define an 0 -quasi-isomorphism from $K X .(\lambda)$ to the complex $X .(\lambda)$, and from (4) we obtain that $X .(\lambda)$ belongs to $A$.

\section{COROllary 2.2. Any O-exact complex is A-exact.}

ProOF. It is sufficient to consider the case when the domain of definition is a polydisk. We have to prove that the class of all $A$-exact complexes satisfies requirements (1)-(4). In order to prove (1), note that the continuous selection theorem allows us to lift any function from $A\left(U, \operatorname{Im} d_{i}\right)$ up to a continuous $X_{i}$ valued function on $U$. Applying to this function the standard projection from the space $C\left(\bar{U}, X_{i}\right)$ of all continuous $X_{i}$-valued functions to the space $A\left(U, X_{i}\right)$ (see $[\mathbf{4}$, Chapter II, §3]), we obtain the necessary lifting. Conditions (2) and (4) can be obtained by a standard diagram chase. To check (2) it is only necessary to control the norms of corresponding elements at each step of the proof.

We shall prove (3), using an induction on the dimension $n$ of the polydisk $D$. When $n=1$, then the considered complex has the form

$$
0 \rightarrow H(D, X) \stackrel{M_{z}-\lambda I}{\mapsto} H(D, X) \stackrel{\Delta(\lambda)}{\rightarrow} X \rightarrow 0
$$

and the requirement can be directly checked. Suppose that (3) is satisfied on the $(n-1)$-dimensional polydisk $D^{\prime}$. Let $z^{\prime}=\left(z_{1}, \ldots, z_{n-1}\right)$ be the coordinate system on $D^{\prime}, M^{\prime}=\left(M_{z_{1}}, \ldots, M_{z_{n-1}}\right)$, and $H^{\prime}=H\left(D^{\prime}, X\right)$. Let $D_{n}$ be a circle in $\mathbf{C}$ with coordinate function $z_{n}$ and let $D=D^{\prime} \times D_{n}$. Note that $H\left(D_{n}, H^{\prime}\right)=H(D, X)$. As above, we have an $A$-exact complex

$$
0 \rightarrow H\left(D_{n}, H^{\prime}\right) \stackrel{M_{z_{n}}-\lambda_{n} I}{\mapsto} H\left(D_{n}, H^{\prime}\right) \stackrel{\Delta\left(\lambda_{n}\right)}{\rightarrow} H^{\prime} \rightarrow 0
$$

This complex induces a bicomplex

$$
0 \rightarrow K \cdot\left(M^{\prime}, H(D, X)\right)(\lambda) \stackrel{M_{z_{n}}-\lambda_{n} I}{\mapsto} K \cdot\left(M^{\prime}, H(D, X)\right)(\lambda) \stackrel{\Delta\left(\lambda_{n}\right)}{\rightarrow} K \cdot\left(M^{\prime}, H^{\prime}\right) \rightarrow \cdots
$$

It is easy to see that the total complex of the bicomplex, formed by the first two columns, is equal to the Koszul complex $K \cdot(M, H(D, X))(\lambda)$. Then from (2) we obtain between the complexes $K \cdot(M, H(D, X))(\lambda)$ and $K \cdot\left(M^{\prime}, H\left(D^{\prime}, X\right)\right)(\lambda)$ an $A$ quasi-isomorphism compatible with the evaluation morphism. Using the inductive assumption and property (4), we obtain that the complex considered in (3) is $A$ exact, which completes the proof.

\section{Parameterized L. Schwartz theorem.}

LEMMA 3.1. Suppose that $X .(\lambda)=\left\{X_{i}, d_{i}(\lambda)\right\}$ and $Y .(\lambda)=\left\{Y_{i}, s_{i}(\lambda)\right\}$ are holomorphic complexes of Fréchet spaces on the domain $D$, and all the spaces $X_{i}$, $Y_{i}$ are inverse limits of Hilbert spaces. Let $K .: X .(\lambda) \rightarrow Y .(\lambda)$ be an O-quasiisomorphism of complexes and all its components $K_{i}: X_{i} \rightarrow Y_{i}$ be compact operators. Then the complexes $X .(\lambda), Y .(\lambda)$ are $\mathcal{O}$-Fredholm on $D$.

We need an intermediate result. 
LEMMA 3.2. Under the hypotheses of Lemma 3.1 suppose that the complexes $X .\left(\lambda_{0}\right), Y \cdot\left(\lambda_{0}\right)$ are exact for some $\lambda_{0}$ at the $(i+1)$ st stage. Then $X .(\lambda), Y \cdot(\lambda)$ are 0 -exact in a neighborhood of $\lambda_{0}$ at the same stage.

ProOF. Fix a compact neighborhood $W$ of $\lambda_{0}$ in $D$. We can suppose that for any $n, i, \lambda \in W$ we have $\left\|d_{i}(\lambda) x\right\|_{n} \leq\|x\|_{n},\left\|s_{i}(\lambda) x\right\|_{n} \leq\|x\|_{n}$, and for any positive $m, n$ there exists a constant $D_{m, n}$ such that for all $i\left\|K_{i} x\right\|_{m} \leq D_{m, n}\|x\|_{n}$. Fix the seminorms $\|\cdot\|_{p}$ on $X_{i}$ and $\|\cdot\|_{r}$ on $Y_{i-1}$. It follows from the $A$-exactness of the cone of $K$. that there exist natural numbers $q, s$ and a constant $C$ such that for any $U \subset W, x(\lambda) \in A\left(U, X_{i+1}\right), y(\lambda) \in A\left(U, Y_{i}\right)$ satisfying $d_{i+1}(\lambda) x(\lambda)$, $s_{i}(\lambda) y(\lambda)=K_{i+1} x(\lambda)$, there exist $x^{\prime}(\lambda) \in A\left(U, X_{i}\right), y^{\prime}(\lambda) \in A\left(U, Y_{i-1}\right)$ such that $d_{i}(\lambda) x^{\prime}(\lambda)=x(\lambda), K_{i} x^{\prime}(\lambda)+s_{i-1}(\lambda) y^{\prime}(\lambda)=y(\lambda)$ and $\left\|x^{\prime}(\lambda)\right\|_{U, p}+\left\|y^{\prime}(\lambda)\right\|_{U, r} \leq$ $C\left(\|x(\lambda)\|_{U, q}+\|y(\lambda)\|_{U, s}\right)$. (We shall suppose that $q \geq p$.) Further, there exist $m, t$ and a constant, denoted again by $C$, such that for any $U \subset D, u(\lambda) \in A\left(U, X_{i+2}\right)$, $v(\lambda) \in A\left(U, Y_{i+1}\right)$, satisfying $d_{i+2}(\lambda) u(\lambda)=0, s_{i+1}(\lambda) v(\lambda)=K_{i+2} u(\lambda)$, there exist $u^{\prime}(\lambda) \in A\left(U, X_{i+1}\right)$ and $v^{\prime}(\lambda) \in A\left(U, Y_{i}\right)$ such that

$$
d_{i+1}(\lambda) u^{\prime}(\lambda)=u(\lambda), \quad K_{i+1} u^{\prime}(\lambda)+s_{i}(\lambda) v^{\prime}(\lambda)=v(\lambda),
$$

and

$$
\left\|u^{\prime}(\lambda)\right\|_{U, q}+\left\|v^{\prime}(\lambda)\right\|_{U, s} \leq C\left(\|u(\lambda)\|_{U, m}+\|v(\lambda)\|_{U, t}\right)
$$

Since the complex $Y \cdot\left(\lambda_{0}\right)$ is exact at $Y_{i+1}$, then, increasing $t$, we can suppose that for any $y$ ker $d_{i+1}\left(\lambda_{0}\right)$ there exists $y^{\prime} \in Y_{i}$ such that $s_{i}\left(\lambda_{0}\right) y^{\prime}=y,\left\|y^{\prime}\right\|_{s} \leq D\|y\|_{t}$ for some constant $D$.

Let $\lambda_{n} \rightarrow \lambda_{0}$ be a sequence of points of $W$ and $x_{n} \in X_{i+1}$ be a sequence of elements such that $d_{i+1}\left(\lambda_{n}\right) x_{n}=0$ and $\left\|x_{n}\right\|_{q} \leq 1$. Replacing the sequence $x_{n}$ by a subsequence, one can find an element $x_{0} \in Y_{i+1}$, such that $K_{i+1} x_{n} \mapsto x_{0}$. Since $s_{i+1}\left(\lambda_{0}\right) x_{0}=\lim s_{i+1}\left(\lambda_{n}\right) \circ K_{i+1} x_{n}=0$, there exists an element $y_{0} \in Y_{i}$ such that $s_{i}\left(\lambda_{0}\right) y_{0}=x_{0},\left\|y_{0}\right\|_{s} \leq D\left\|x_{0}\right\|_{t} \leq D . D_{t, q}$. Now $s_{i}\left(\lambda_{n}\right) y_{0}-K_{i+1} x_{n} \rightarrow 0$ and therefore there exist sequences $z_{n} \in X_{i}, u_{n} \in Y_{i+1}$ such that

$$
d_{i}\left(\lambda_{n}\right) z_{n}-x_{n} \mapsto 0, \quad K_{i} z_{n}+s_{i-1}\left(\lambda_{n}\right) u_{n} \mapsto y_{0}
$$

and

$$
\left\|z_{n}\right\|_{p}+\left\|u_{n}\right\|_{r} \leq C^{\prime} C\left(1+D \cdot D_{t, q}\right) .
$$

Fix $\varepsilon>0$. Since the sequence $x_{n}$ can be chosen arbitrarily and the constant $C^{\prime}$ does not depend on the choice, one can find a neighborhood $V=V(\varepsilon)$ of $\lambda_{0}$ such that for any $\lambda \in V$ and any $x \in \operatorname{ker} d_{i+1}(\lambda)$ with $\|x\|_{q} \leq 1$, there exists an element $z \in X$ satisfying $\|z\|_{p} \leq C^{\prime}$ and $\left\|x-d_{i}(\lambda) z\right\|_{q}<\varepsilon$.

Now we need to find a canonical choice of an element $z$. Using the compactness of $K_{i}$, we can choose a finite-dimensional subspace $H$ of $X_{i}$ such that for any $z \in X_{i},\|z\|_{p} \leq 1$, there exists $\tilde{z} \in H$ such that $\|\tilde{z}\|_{p} \leq 1,\left\|K_{i} \tilde{z}-K_{i} z\right\|_{t} \leq \varepsilon / C^{\prime}$. Let $H(\lambda)=s_{i}(\lambda) \circ K_{i}[H]$. We can choose $H$ such that $K_{i} H \cap \operatorname{ker} s_{i}\left(\lambda_{0}\right)=0$, and therefore, near $\lambda_{0}, H(\lambda)$ is a finite-dimensional subspace of $Y_{i+1}$ of constant dimension. For any $\lambda \in V, x \in \operatorname{ker} d_{i+1}(\lambda),\|x\|_{q} \leq 1$ there exists $z \in H,\|z\|_{p} \leq C^{\prime}$, such that $\left\|s_{i}(\lambda) \circ K_{i} z-K_{i+1} x\right\|_{t} \leq 2 \varepsilon$. Denote by $P(\lambda)$ the projector from $Y_{i+1}$ to $H(\lambda)$ such that ker $P(\lambda)=L$, where $L$ is the orthogonal complement of $H\left(\lambda_{0}\right)$ with respect to the scalar product corresponding to $\|\cdot\|_{t}$. We can find a sufficiently small neighborhood $V^{\prime}(\varepsilon)$ of $\lambda_{0}$ such that for any $\lambda \in V^{\prime}$ and $y \in Y_{i+1}$ we have 
$\left\|y-P(\lambda) y_{t}\right\| \leq 2 d_{t}(y, H(\lambda))$, where $d_{t}$ is the pseudodistance determined by $\|\cdot\|_{t}$. Then for any $\lambda \in V^{\prime}, x \in \operatorname{ker} d_{i+1}(\lambda)$ with $\|x\|_{q} \leq 1$, we have

$$
\left\|K_{i+1} x-P(\lambda) \circ K_{i+1} x\right\|_{t} \leq 4 \varepsilon
$$

and

$$
P(\lambda) \circ K_{i+1} x=s_{i}(\lambda) \circ K_{i} z
$$

for some $z \in H,\|z\|_{p} \leq 2 C^{\prime}$.

Let $U$ be an arbitrary polydisk contained in $V(\varepsilon) \cap V^{\prime}(\varepsilon)$, and let $x(\lambda)$ be an element of $A\left(U, X_{i+1}\right)$ such that $d_{i+1}(\lambda) x(\lambda)=0$ and $\|x(\lambda)\|_{U, q} \leq 1$. Take $z(\lambda) \in$ $A(U, H)$ such that $s_{i}(\lambda) \circ K_{i} z(\lambda)=P(\lambda) \circ K_{i+1} x(\lambda)$. Then $\|z(\lambda)\|_{U, p} \leq 2 C^{\prime}$ and $\left\|s_{i}(\lambda) \circ K_{i} z(\lambda)-K_{i+1} x(\lambda)\right\|_{t} \leq 4 \varepsilon$. Using the $A$-exactness of the cone of $K$., we can find $u(\lambda) \in A\left(U, Y_{i}\right)$ and $v(\lambda) \in A\left(U, X_{i+1}\right)$ such that $s_{i}(\lambda) u(\lambda)-K_{i+1} v(\lambda)=$ $s_{i}(\lambda) \circ K_{i} z(\lambda)-K_{i+1} x(\lambda)$ and $\|u(\lambda)\|_{U, s}+\|v(\lambda)\|_{U, q} \leq 4 C \varepsilon$. Further, there exist $y(\lambda) \in A\left(U, X_{i}\right)$ and $w(\lambda) \in A\left(U, Y_{i-1}\right)$ such that $d_{i}(\lambda) y(\lambda)=x(\lambda)-v(\lambda), K_{i} y(\lambda)-$ $s_{i-1}(\lambda) w(\lambda)=K_{i} z(\lambda)-u(\lambda)$, and $\|y(\lambda)\|_{U, p} \leq C^{\prime \prime}=2 C^{\prime} D_{p, s}+8 C+1$. We have $\left\|d_{i}(\lambda) y(\lambda)-x(\lambda)\right\|_{U, q} \leq 4 C \varepsilon$. Now take $\varepsilon$ such that $4 C \varepsilon<1 / 2$. Iterating the procedure, we obtain a sequence of vector-functions $y_{n}(\lambda) \in A\left(U, X_{i}\right)$ such that

$$
\sum_{n=1}^{\infty}\left\|y_{n}(\lambda)\right\|_{U, p} \leq 2 C^{\prime \prime}
$$

and

$$
\left\|d_{i}(\lambda)\left[\sum_{n=1}^{N} y_{n}(\lambda)\right]-x(\lambda)\right\|_{U, q} \leq 2^{-N} .
$$

The series $\sum_{n=1}^{\infty} K_{i} y_{n}(\lambda)$ is converging in $A\left(U, Y_{i}\right)$ to some element $\tilde{y}(\lambda) \in A\left(U, Y_{i}\right)$, and it is easy to see that $s_{i}(\lambda) \tilde{y}(\lambda)=K_{i+1} x(\lambda)$. Using again the exactness of the cone, we obtain $y(\lambda) \in A\left(U, X_{i}\right)$ such that $d_{i}(\lambda) y(\lambda)=x(\lambda)$. Lemma 3.2 is proved.

ProOF OF LEMMA 3.1. Let $\lambda_{0} \in D$. We shall construct on some neighborhood of $\lambda_{0}$ a holomorphic complex $H .(\lambda)=\left\{H_{i}, a_{i}(\lambda)\right\}$ of finite-dimensional spaces, and an 0 -quasi-isomorphism $\varphi \cdot(\lambda): H .(\lambda) \rightarrow X .(\lambda)$. Suppose that $H_{i}, a_{i}(\lambda)$, and $\varphi_{i}(\lambda)$ are already constructed for $i \geq k$, and denote by $\tilde{X} \cdot(\lambda)=\left\{\tilde{X}_{i}, \tilde{d}_{i}(\lambda)\right\}$ the cone of the morphism $\varphi \cdot(\lambda)$, determined in degrees $\geq k$. Since the complex $X \cdot\left(\lambda_{0}\right)$ is Fredholm, the space $\operatorname{ker} \tilde{d}_{k}\left(\lambda_{0}\right) / \operatorname{im} d_{k-1}\left(\lambda_{0}\right)$ is finite dimensional, and we can choose elements $x_{1}, \ldots, x_{m} \in \operatorname{ker} \tilde{d}_{k}\left(\lambda_{0}\right)$ which generate this space. One can find a seminorm $\|\cdot\|_{p}$ and $\varepsilon>0$ such that any other system $x_{1}^{\prime}, \ldots, x_{m}^{\prime} \in \operatorname{ker} \tilde{d}_{k}\left(\lambda_{0}\right)$, satisfying $\left\|x_{i}^{\prime}-x_{i}\right\|_{p}<\varepsilon, i=1, \ldots, m$, generates the space of homologies also. The complex $\tilde{X} .(\lambda)$ is $A$-exact at $X_{k+1}$, and we can choose a constant $C$ and a number $q$ such that for any sufficiently small polydisk $U$ containing $\lambda_{0}$ and any vector-function $x(\lambda) \in A\left(U, X_{k+1}\right)$ satisfying $d_{k+1}(\lambda) x(\lambda)=0$, there exists $y(\lambda) \in A\left(U, X_{k}\right)$ with $d_{k}(\lambda) y(\lambda)=x(\lambda),\|y(\lambda)\|_{U, p} \leq C\|x(\lambda)\|_{U, q}$. We can take a sufficiently small $U$ such that $\left\|d_{k}(\lambda) x_{i}\right\|_{U, q} \leq \varepsilon / C, i=1, \ldots, m$. Then there exist vector-functions $y_{1}(\lambda), \ldots, y_{m}(\lambda) \in A(U, X)$ such that $d_{k}(\lambda) y_{i}(\lambda)=d_{k}(\lambda) x_{i}$ and $\left\|y_{i}(\lambda)\right\|_{U, p} \leq$ $\varepsilon, i=1, \ldots, m$. Put $x_{i}(\lambda)=x_{i}-y_{i}(\lambda)$. Then $x_{1}\left(\lambda_{0}\right), \ldots, x_{m}\left(\lambda_{0}\right)$ generate $\operatorname{ker} \tilde{d}_{k}\left(\lambda_{0}\right) / \operatorname{im} d_{k-1}\left(\lambda_{0}\right)$ and $d_{k}(\lambda) x_{i}(\lambda)=0, i=1, \ldots, m$. Setting $H_{k}=\mathbf{C}^{m}$, denote by $x(\lambda)$ the map from $H_{k}$ to $\tilde{X}_{k}$ determined by the functions $x_{1}(\lambda), \ldots, x_{m}(\lambda)$, and let $a_{k}(\lambda), \varphi_{k}(\lambda)$ be the projections of $x(\lambda)$ on $H_{k+1}$ and $X_{k}$. Now the complex 
$H .(\lambda)=\left\{H_{i}, a_{i}(\lambda)\right\}$ and the morphism of complexes $\varphi \cdot(\lambda)=\left\{\varphi_{i}(\lambda)\right\}$ are defined also for $i=k$, and $\varphi \cdot\left(\lambda_{0}\right)$ is a quasi-isomorphism. Let $\psi \cdot(\lambda)=K \cdot \circ \varepsilon \cdot(\lambda)$, and denote by $\tilde{Y} \cdot(\lambda)$ the cone of the morphism $\psi$. Let $\tilde{K} .: \tilde{X} .(\lambda) \rightarrow \tilde{Y} \cdot(\lambda)$ be the morphism of complexes determined by the morphism $K$. and the identity map of $H \cdot(\lambda)$. Since $K$. is an $O$-quasi-isomorphism, so is $\tilde{K}$. Applying Lemma 3.2, we obtain that $\tilde{X}$. $(\lambda)$ is $O$-exact; i.e., $\varphi .(\lambda)$ is an 0 -quasi-isomorphism. Lemma 3.1 is proved.

Applying Lemma 3.1 to the resolution of the direct image of a sheaf, constructed in $\S 1$, we complete the proof of the Grauert theorem.

4. Remark. The only choice involved in the construction of the resolution in $\S 1$ is that of the coordinate system on the base. Due to this, the construction has the following functorial properties.

(1) The resolution $K C \cdot(S, \mathcal{U}, \mathcal{L})(\lambda)$ is an exact functor of $\mathcal{L}$.

(2) If $\varphi: U \rightarrow U^{\prime}$ is a holomorphic mapping of the base domain, proper on the support of $f_{*} \mathcal{L}$, and if $K C^{\prime}(S, \mathcal{U}, \mathcal{L})(\lambda)$ is the Koszul resolution of the direct image of $\mathcal{L}$ under the map $\varphi \circ f$, then there exists a natural morphism of $K C \cdot(S, \mathcal{U}, \mathcal{L})(\lambda)$ in $\varphi^{*} K C^{\prime}(S, \mathcal{U}, \mathcal{L})(\lambda)$.

\section{REFERENCES}

1. H. Grauert, Ein Theorem der analytischen Garbentheorie und die Modulräume Complexer Structuren, Inst. Hautes Études Sci. Publ. Math. no. 5 (1960).

2. O. Forster and K. Knorr, Ein beweis des Grauertschen Bildgarbensatzes nach Ideen von B. Malgrange, Manuscripta Math. 5 (1971), 19-44.

3. J. L. Taylor, A joint spectrum for several commuting operators, J. Funct. Anal. 6 (1970), $1-38$.

4. H. Grauert and R. Remmert, Theory of Stein spaces, Springer-Verlag, 1979.

Mathematical Institute of the Bulgarian ACADEMy of SCIEnCES, Sofia, BulGARIA

Current address: Faculty of Mathematics, Softa State University, B-d "A. Ivanov" 5, Sofia 1126, Bulgaria 